\title{
Causality in acceleration radiation
}

\author{
Marlan O. Scully, ${ }^{1,2,3}$ Anatoly A. Svidzinsky $\odot,{ }^{1}$ and William Unruh ${ }^{4}$ \\ ${ }^{1}$ Texas A\&M University, College Station, Texas 77843, USA \\ ${ }^{2}$ Baylor University, Waco, Texas 76798, USA \\ ${ }^{3}$ Princeton University, Princeton, New Jersey 08544, USA \\ ${ }^{4}$ University of British Columbia, Vancouver, Canada V6T 121
}

(Received 20 May 2019; published 20 November 2019)

\begin{abstract}
The character of acceleration radiation is analyzed by considering the joint probability of an accelerated atom emitting a photon and a photodetector fixed in space registering a count. This simple model yields insight into counterintuitive issues associated with causality, vacuum entanglement, and related topics.
\end{abstract}

DOI: 10.1103/PhysRevResearch.1.033115

\section{INTRODUCTION}

Acceleration radiation emitted by neutral atoms undergoing uniform acceleration is a rich but somewhat subtle problem. Indeed R. Feynman had the note on his final blackboard (see, e.g., Fig. 1 in Ref. [1]) listing "acceleration temperature" as something "to learn!" One of the early inroads into the subject came from the $1969 \mathrm{Ph}$. D. thesis of optical physicist G. Moore [2], who showed that an accelerating mirror will generate photons. This stimulated S. Fulling [3] to do his Ph. D. thesis on "Quantum Field Theory in Curved Space-Time," showing that the vacuum in a stationary (Minkowski) frame will look very different in an accelerating (Rindler) frame. This "quantum fields in curved space-time perspective" was the basis of S. Hawking's famous demonstration that a black hole can emit thermal radiation [4,5]. W. Unruh [6] then used the same formalism to show that atoms undergoing constant acceleration " $a$ " will be excited as if they are in a bath of thermal photons having temperature $T_{U}=\hbar a / 2 \pi c k_{B}$, where $c$ is the speed of light and $k_{B}$ is the Boltzmann constant.

From a quantum optical perspective [7-9] the acceleration radiation, also known as Unruh radiation, derives from the counter-rotating terms in the interaction Hamiltonian given by

$$
\hat{V}_{\text {counter-rotating }} \Rightarrow \hat{\sigma}^{+} \hat{a}_{\mathbf{k}}^{\dagger} e^{i\left(\omega+v_{k}\right) t},
$$

which represents the virtual process in which an atom "jumps" to the excited state while emitting a photon. In Eq. (1), $\hat{\sigma}^{+}$is raising operator for the atom with transition angular frequency $\omega$ and $\hat{a}_{\mathbf{k}}^{\dagger}$ is creation operator of a photon with wave vector $\mathbf{k}$ and angular frequency $v_{k}$. However, this excitation-emission process can be made real when the atom undergoes acceleration. In other words, the usual virtual process, in which emission is rapidly followed by reabsorption, is actuated by

Published by the American Physical Society under the terms of the Creative Commons Attribution 4.0 International license. Further distribution of this work must maintain attribution to the author(s) and the published article's title, journal citation, and DOI. breaking adiabaticity (due to acceleration) which leaves the atom and the radiation oscillator excited.

For a uniformly accelerating observer, the background appears to be in thermodynamic equilibrium with temperature $T_{U}$ and the atom gets excited by absorbing a thermal photon. The atomic excitation associated with the thermal bath of acceleration radiation involves the $\hat{\sigma}^{+} \hat{a}_{\mathbf{k}}$ terms in the Hamiltonian. These two points of view: the Minkowski space $\hat{\sigma}^{+} \hat{a}_{\mathbf{k}}^{\dagger}$ approach and the Rindler space (noninertial frame) $\hat{\sigma}^{+} \hat{a}_{\mathbf{k}}$ approach are complementary. The interplay between these two vantage points can be subtle [10] and is the subject of this and two following papers as is discussed in the conclusion.

In particular, we are interested in the question of the emission of radiation from atom 1 which is undergoing constant acceleration $a$ and propagation of this radiation so as to excite a fixed atom 2 (see Fig. 1). We assume that atom 2 is located to the left from atom 1 . The interaction between the two atoms is somewhat subtle. For example, under some conditions, right running waves of atom 1 can strongly contribute to the probability of finding radiation on the left-hand side of the vertical time axis $(z<0)$ [10]. However, in the present paper, we show that for a stationary detector atom 2 exposed to radiation from atom 1 (from $t=-\infty$ to $t=\infty$ ) only the left running modes contribute.

\section{JOINT EXCITATION OF TWO ATOMS}

The atom-field interaction Hamiltonian $\hat{V}(t)=\hat{V}_{1}(t)+$ $\hat{V}_{2}(t)$ is given by

$$
\begin{aligned}
\hat{V}(t)= & \wp_{1}\left(\hat{\sigma}_{1} e^{-i \omega_{1} \tau(t)}+\hat{\sigma}_{1}^{\dagger} e^{i \omega_{1} \tau(t)}\right) \hat{E}\left(t, z_{1}(t)\right) \\
& +\wp_{2}\left(\hat{\sigma}_{2} e^{-i \omega_{2} t}+\hat{\sigma}_{2}^{\dagger} e^{i \omega_{2} t}\right) \hat{E}\left(t, z_{2}\right),
\end{aligned}
$$

where $\wp_{j}$ is the dipole matrix element for the $j$ th atom $(j=1,2), \hat{\sigma}_{j}$ is the atomic lowering operator, $\hat{\sigma}_{j}^{\dagger}$ is the corresponding raising operator, and $\tau(t)$ is the proper time of the accelerated atom 1 which moves along trajectory $z_{1}(t)$. In this paper, we consider either dimension $1+1$, or dimension $3+1$ but restrict photons to have wave vector $\mathbf{k}$ parallel to the $z$ axis. The electric field operator at spatial coordinate $z_{j}$ is 
Minkowski space

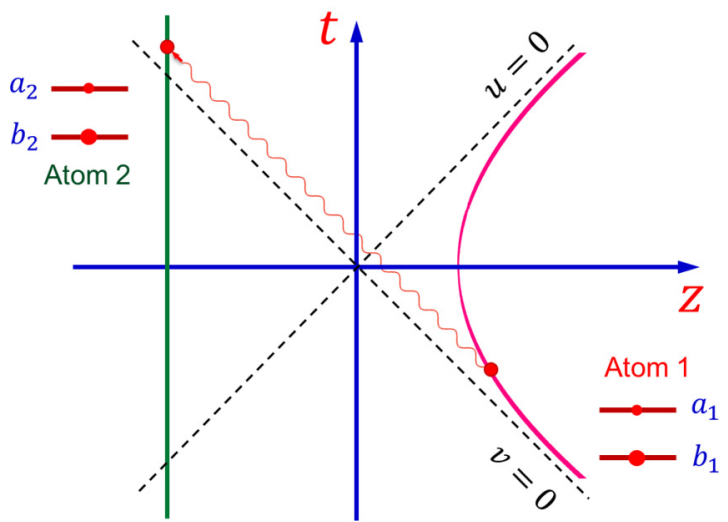

FIG. 1. Atom 1 accelerates from $-\infty$ to $+\infty$ along hyperbolic trajectory where the null geodesics $u=t-\frac{z}{c}=0$ and $v=t+\frac{z}{c}=0$ are indicated by dashed lines. Unruh acceleration radiation from atom 1 is shown as a wavy line which is absorbed by the detector atom 2; atomic states $\left|a_{j}\right\rangle$ and $\left|b_{j}\right\rangle$ are shown as weakly excited.

given by

$$
\hat{E}\left(t, z_{j}\right)=-\frac{\partial \hat{A}\left(t, z_{j}\right)}{\partial t}=\sum_{k} \mathcal{E}_{k} \hat{a}_{k} e^{-i \nu_{k}\left(t-\kappa z_{j} / c\right)}+a d j,
$$

where $\hat{A}(t, z)$ is the vector potential operator, $\kappa$ is the "unit vector" in $2 \mathrm{D}$ (one time + one space dimension) such that $\kappa=+1$ for right propagating photons and $\kappa=-1$ for left propagation, $\mathcal{E}_{k}=\sqrt{\hbar v_{k} / 2 \epsilon_{0} V_{\mathrm{ph}}}$ is the electric field per photon of frequency $v_{k}=c k, V_{\mathrm{ph}}$ is the quantization volume [11], and $\hat{a}_{k}$ is the annihilation operator for a photon of wave vector $k$.

In the second order in the coupling constant, the probability amplitude that both the fixed detector atom 2 and the accelerated atom 1 get excited, and the field is in the vacuum state $|0\rangle$, is given by

$$
\begin{aligned}
\mathcal{A} & =\left\langle a_{1} a_{2} 0|\hat{U}(t)| b_{1} b_{2} 0\right\rangle \\
& \cong-\frac{1}{\hbar^{2}}\left\langle a_{1} a_{2} 0\left|\int_{t_{0}}^{t} d t^{\prime} \int_{t_{0}}^{t^{\prime}} d t^{\prime \prime} \hat{V}\left(t^{\prime}\right) \hat{V}\left(t^{\prime \prime}\right)\right| b_{1} b_{2} 0\right\rangle,
\end{aligned}
$$

which is usefully written in terms of the commutators [,] and anticommutators $\{$,$\} as$

$$
\begin{aligned}
\mathcal{A}= & -\frac{1}{2 \hbar^{2}} \int_{t_{0}}^{t} d t^{\prime} \int_{t_{0}}^{t^{\prime}} d t^{\prime \prime} \\
& \times\left\langle a_{1} a_{2} 0\left|\left(\left[\hat{V}\left(t^{\prime}\right), \hat{V}\left(t^{\prime \prime}\right)\right]+\left\{\hat{V}\left(t^{\prime}\right), \hat{V}\left(t^{\prime \prime}\right)\right\}\right)\right| b_{1} b_{2} 0\right\rangle .
\end{aligned}
$$

As is shown in Appendix A, in the long time limit of $t_{0} \rightarrow-\infty$ and $t \rightarrow \infty$, Eq. (3) becomes

$$
\mathcal{A}=-\frac{\wp_{1} \wp_{2} \omega_{2}}{4 \epsilon_{0} \hbar c A_{\mathrm{ph}}} \int_{-\infty}^{\infty} d t^{\prime} \sum_{i=1}^{4} K_{i}\left(t^{\prime}, z_{1}\left(t^{\prime}\right), z_{2}\right)
$$

where $A_{\text {ph }}$ is the photon quantization area. The kernels $K_{i}$ are given in Table I, where it is seen that contributions from the right propagating photons differ in sign and cancel out. This is what we would expect since the detector atom 2 is placed to
TABLE I. Kernels for commutator and anticommutator terms. Here, $z_{1}^{\prime}=z_{1}\left(t^{\prime}\right)$.

\begin{tabular}{lcc}
\hline \hline & Kernel & Origin \\
\hline$K_{1}$ & $e^{i\left(\omega_{1} \tau\left(t^{\prime}\right)+\omega_{2} t^{\prime}\right)-i \omega_{2}\left(z_{1}^{\prime}-z_{2}\right) / c}$ & $\begin{array}{c}\text { anticommutator } \\
\text { right propagator }\end{array}$ \\
$K_{2}$ & $e^{i\left(\omega_{1} \tau\left(t^{\prime}\right)+\omega_{2} t^{\prime}\right)+i \omega_{2}\left(z_{1}^{\prime}-z_{2}\right) / c}$ & anticommutator \\
& & left propagator \\
$K_{3}$ & $e^{i\left(\omega_{1} \tau\left(t^{\prime}\right)+\omega_{2} t^{\prime}\right)+i \omega_{2}\left(z_{1}^{\prime}-z_{2}\right) / c}$ & commutator \\
& & left propagator \\
$K_{4}$ & $-e^{i\left(\omega_{1} \tau\left(t^{\prime}\right)+\omega_{2} t^{\prime}\right)-i \omega_{2}\left(z_{1}^{\prime}-z_{2}\right) / c}$ & commutator \\
& & right propagator \\
\hline \hline
\end{tabular}

the left of the radiating atom 1 so that only the left propagating modes can excite the detector atom. The net result is

$$
\mathcal{A}=-\frac{\wp_{1} \wp_{2} \omega_{2}}{2 \epsilon_{0} \hbar c A_{\mathrm{ph}}} \int_{-\infty}^{\infty} d t^{\prime} e^{i\left(\omega_{1} \tau\left(t^{\prime}\right)+\omega_{2} t^{\prime}\right)+i \omega_{2}\left(z_{1}\left(t^{\prime}\right)-z_{2}\right) / c} .
$$

Equation (5) is recognized as the integral appearing in, for example, earlier work [9] in which the atomic frequency $\omega$ and the radiation frequency $v$ coming from the $\hat{\sigma}_{1}^{\dagger}(\tau) \hat{a}_{k}^{\dagger} e^{i v_{k}\left(t(\tau)-\kappa z_{1}(\tau) / c\right)}$ terms of Eq. (2), where

$$
\begin{aligned}
t(\tau) & =\frac{c}{a} \sinh \left(\frac{a \tau}{c}\right), \\
z_{1}(\tau) & =\frac{c^{2}}{a} \cosh \left(\frac{a \tau}{c}\right)
\end{aligned}
$$

is the trajectory of the uniformly accelerated atom 1 with acceleration $a$. An alternative derivation of Eq. (5) based on coordinate transformations $u=t-z / c$ and $v=t+z / c$ has some advantages and is given in Appendix B.

Carrying out the integral in Eq. (5) and squaring we find the joint probability of both atoms being excited and field in the Minkowski vacuum state to be

$$
P_{a_{1} a_{2} 0} \cong \frac{\pi \wp_{1}^{2} \wp_{2}^{2} \omega_{2}^{2}}{8 \epsilon_{0}^{2} \hbar^{2} c a A_{\mathrm{ph}}^{2} \omega_{1}} \frac{1}{e^{\frac{2 \pi c \omega_{1}}{a}}-1} .
$$

\section{CONCLUSIONS}

The joint probability that both the emitter (atom 1) and detector (atom 2) are excited during the time from $-\infty$ to $+\infty$ and the field remains in the Minkowski vacuum state goes as the Planck factor with the Unruh temperature (average Unruh photon number). Note that the emission of radiation associated with the counter propagating term goes as $\left\langle\hat{a} \hat{a}^{\dagger}\right\rangle=n+1$ whereas the absorption goes as $\left\langle\hat{a}^{\dagger} \hat{a}\right\rangle=n$ so that together the total probability of both atoms being excited would be expected to go as $(n+1) n$. Since $n \ll 1$, we find the result of Eq. (7) to be physically correct.

It is interesting to note that only the left propagating modes contribute to the joint excitation probability given by Eq. (7). While it might seem that this is intuitively obvious in the sense that the light from the accelerated atom propagates to the left and right, but only the left propagating mode would excite the left laying detector atom, the detailed calculation in Appendix B shows that this interpretation is naive. It just so happens that the entanglement of the quantum fields from 
the right moving modes (as seen in the anticommutator terms in Appendix B) cancel with the delta function terms in the commutator, and add for the left moving modes. There is a subtle interplay of entanglement and causal change which leads to the results we see.

Thus while it might seem obvious that only the left propagating modes would contribute, as seen from Table I, this could lead to a bad intuition in cases where the stationary detector had a time dependent interaction with the fields (such as that studied by Unruh and Wald [10]). It is thus important to note that different detection situations can lead to very different results. In particular, the present simple model in which the detector atom has two sharp levels and is left in place for the entire duration of the emission process associated with the atom 1 gives the result of Eq. (7). Whereas a model in which we turn the detector atom on at some time $T$ and off at $T+\Delta T$ can lead to very different results; namely, the joint probability of both atoms being excited now shows that the probability of excitation at the specific time $T$ involves the noncausal region $z<c|t|$ due to the entanglement terms as expressed by the anticommutator. We emphasize that the sharp single frequency model leading to the joint probability given by Eq. (7) comes from the left propagating modes. The sharp time, broad frequency, model also involves the right propagating modes, and is not causal. We will return to this and other related issues in a later paper.

\section{ACKNOWLEDGMENTS}

This work was supported by the Air Force Office of Scientific Research (Award FA9550-18-1-0141), the Office of Naval Research (Awards N00014-16-1-3054 and N0001416-1-2578), the Robert A. Welch Foundation (Award A1261), and the Natural Sciences and Engineering Research Council of Canada (NSERC). W.U. thanks the Hagler Institute for Advanced Studies, the Canadian Institute for Advanced Research, the Alexander von Humboldt Foundation and Helmholtz Association for their support. We also thank J. Ben-Benjamin, M. Kim, R. Nessler, and Z. Zhang for their useful discussions.

\section{APPENDIX A: CALCULATION OF EXCITATION PROBABILITY}

Starting with the expression for the evolution operator $\hat{U}$

$$
\hat{U}(t) \cong 1-\frac{i}{\hbar} \int_{t_{0}}^{t} \hat{V}\left(t^{\prime}\right) d t^{\prime}-\frac{1}{\hbar^{2}} \int_{t_{0}}^{t} d t^{\prime} \int_{t_{0}}^{t^{\prime}} d t^{\prime \prime} \hat{V}\left(t^{\prime}\right) \hat{V}\left(t^{\prime \prime}\right)+\cdots,
$$

we may write the second-order term as

$$
-\frac{1}{2 \hbar^{2}} \int_{t_{0}}^{t} d t^{\prime} \int_{t_{0}}^{t^{\prime}} d t^{\prime \prime}\left(\left\{\hat{V}\left(t^{\prime}\right) \hat{V}\left(t^{\prime \prime}\right)+\hat{V}\left(t^{\prime \prime}\right) \hat{V}\left(t^{\prime}\right)\right\}+\left[\hat{V}\left(t^{\prime}\right) \hat{V}\left(t^{\prime \prime}\right)-\hat{V}\left(t^{\prime \prime}\right) \hat{V}\left(t^{\prime}\right)\right]\right) .
$$

The \{\} anticommutator term may be written as

$$
\int_{t_{0}}^{t} d t^{\prime} \int_{t_{0}}^{t^{\prime}} d t^{\prime \prime}\left\{\hat{V}\left(t^{\prime}\right), \hat{V}\left(t^{\prime \prime}\right)\right\}=\left[\int_{t_{0}}^{t} d t^{\prime} \int_{t_{0}}^{t^{\prime}} d t^{\prime \prime}+\int_{t_{0}}^{t} d t^{\prime} \int_{t^{\prime}}^{t} d t^{\prime \prime}\right] \hat{V}\left(t^{\prime}\right) \hat{V}\left(t^{\prime \prime}\right)=\int_{t_{0}}^{t} d t^{\prime} \int_{t_{0}}^{t} d t^{\prime \prime} \hat{V}\left(t^{\prime}\right) \hat{V}\left(t^{\prime \prime}\right) .
$$

Using $\hat{V}(t)=\hat{V}_{1}(t)+\hat{V}_{2}(t)$ together with Eqs. (A2) and (A3), we have the second-order approximation to the excitation probability amplitude

$$
\left\langle a_{1} a_{2} 0|\hat{U}(t)| b_{1} b_{2} 0\right\rangle=-\frac{1}{2 \hbar^{2}}\left\langle a_{1} a_{2} 0\left|\int_{t_{0}}^{t} d t^{\prime} \int_{t_{0}}^{t} d t^{\prime \prime} \hat{V}_{1}\left(t^{\prime}\right) \hat{V}_{2}\left(t^{\prime \prime}\right)+\int_{t_{0}}^{t} d t^{\prime} \int_{t_{0}}^{t^{\prime}} d t^{\prime \prime}\left[\hat{V}_{1}\left(t^{\prime}\right), \hat{V}_{2}\left(t^{\prime \prime}\right)\right]+(1 \leftrightarrow 2)\right| b_{1} b_{2} 0\right\rangle
$$

Next we calculate the terms in Eq. (A4).

\section{Anticommutator}

$$
\begin{aligned}
\mathcal{A}_{\mathrm{anti}}= & -\frac{1}{2 \hbar^{2}} \int_{-\infty}^{\infty} d t^{\prime} \int_{-\infty}^{\infty} d t^{\prime \prime}\left\langle a_{1} a_{2} 0\left|\hat{V}_{1}\left(t^{\prime}\right) \hat{V}_{2}\left(t^{\prime \prime}\right)+\hat{V}_{2}\left(t^{\prime}\right) \hat{V}_{1}\left(t^{\prime \prime}\right)\right| b_{1} b_{2} 0\right\rangle \\
= & -\frac{\wp_{1} \wp_{2}}{2 \hbar^{2}} \int_{-\infty}^{\infty} d t^{\prime} \int_{-\infty}^{\infty} d t^{\prime \prime}\left[e^{i \omega_{1} \tau\left(t^{\prime}\right)} e^{i \omega_{2} t^{\prime \prime}} \sum_{k} \mathcal{E}_{k}^{2} e^{-i v_{k}\left(t^{\prime}-z_{1}^{\prime} / c\right)+i v_{k}\left(t^{\prime \prime}-z_{2}^{\prime \prime} / c\right)}\right. \\
& \left.+e^{i \omega_{2} t^{\prime}+i \omega_{1} \tau\left(t^{\prime \prime}\right)} \sum_{k} \mathcal{E}_{k}^{2} e^{-i v_{k}\left(t^{\prime}-z_{2}^{\prime} / c\right)+i v_{k}\left(t^{\prime \prime}-z_{1}^{\prime \prime} / c\right)}\right]_{\operatorname{right}(\kappa=1)} \\
& -\frac{\wp_{1} \wp_{2}}{2 \hbar^{2}} \int_{-\infty}^{\infty} d t^{\prime} \int_{-\infty}^{\infty} d t^{\prime \prime}\left[e^{i \omega_{1} \tau\left(t^{\prime}\right)} e^{i \omega_{2} t^{\prime \prime}} \sum_{k} \mathcal{E}_{k}^{2} e^{-i v_{k}\left(t^{\prime}+z_{1}^{\prime} / c\right)+i v_{k}\left(t^{\prime \prime}+z_{2}^{\prime \prime} / c\right)}\right. \\
& \left.+e^{i \omega_{2} t^{\prime}+i \omega_{1} \tau\left(t^{\prime \prime}\right)} \sum_{k} \mathcal{E}_{k}^{2} e^{-i v_{k}\left(t^{\prime}+z_{2}^{\prime} / c\right)+i v_{k}\left(t^{\prime \prime}+z_{1}^{\prime \prime} / c\right)}\right]_{\text {left }(\kappa=-1)}
\end{aligned}
$$


The terms (i) and (iii) after integration over $t^{\prime \prime}$ yield $\delta\left(\omega_{2}+v_{k}\right)$. The argument of the delta function is always positive and, therefore, the terms (i) and (iii) give zero contribution. Integration of other two terms over $t^{\prime}$ gives

$$
\begin{aligned}
\mathcal{A}_{\text {anti }}= & -\frac{\pi \wp_{1} \wp_{2}}{\hbar^{2}} \int_{-\infty}^{\infty} d t^{\prime \prime} e^{i \omega_{1} \tau\left(t^{\prime \prime}\right)} \sum_{k} \mathcal{E}_{k}^{2}\left[\delta\left(\omega_{2}-v_{k}\right) e^{i \nu_{k}\left(t^{\prime \prime}-z_{1}^{\prime \prime} / c\right)} e^{i \nu_{k} z_{2} / c}\right. \\
& \left.+\delta\left(\omega_{2}-v_{k}\right) e^{i \nu_{k}\left(t^{\prime \prime}+z_{1}^{\prime \prime} / c\right)} e^{-i v_{k} z_{2} / c}\right] .
\end{aligned}
$$

We replace $\sum_{k} \rightarrow \frac{L}{2 \pi} \int d k$

$$
\begin{aligned}
\mathcal{A}_{\mathrm{anti}}= & -\frac{\wp_{1} \wp_{2}}{4 \hbar^{2} c} \frac{\hbar \omega_{2}}{\epsilon_{0} A_{\mathrm{ph}}} \int_{-\infty}^{\infty} d t^{\prime}\left[e^{i \omega_{1} \tau\left(t^{\prime}\right)} e^{i \omega_{2}\left(t^{\prime}-z_{1}^{\prime} / c\right)} e^{i \omega_{2} z_{2} / c}\right. \\
& \left.+e^{i \omega_{1} \tau\left(t^{\prime}\right)} e^{i \omega_{2}\left(t^{\prime}+z_{1}^{\prime} / c\right)} e^{-i \omega_{2} z_{2} / c}\right] \\
= & -\frac{\wp_{1} \wp_{2}}{4 \hbar^{2} c} \frac{\hbar \omega_{2}}{\epsilon_{0} A_{\mathrm{ph}}} \int_{-\infty}^{\infty} d t^{\prime}\left[\begin{array}{c}
e^{i\left(\omega_{1} \tau\left(t^{\prime}\right)+\omega_{2} t^{\prime}\right)-i \omega_{2}\left(z_{1}^{\prime}-z_{2}\right) / c}+e^{i\left(\omega_{1} \tau\left(t^{\prime}\right)+\omega_{2} t^{\prime}\right)+i \omega_{2}\left(z_{1}^{\prime}-z_{2}\right) / c} \\
\text { right }
\end{array}\right],
\end{aligned}
$$

where $A_{\mathrm{ph}}=V_{\mathrm{ph}} / L$.

\section{Commutator}

$$
\begin{aligned}
\mathcal{A}_{\mathrm{com}} & =-\frac{1}{2 \hbar^{2}} \int_{t_{0}}^{t} d t^{\prime} \int_{t_{0}}^{t^{\prime}} d t^{\prime \prime}\left\langle a_{1} a_{2} 0\left|\left[\hat{V}_{1}\left(t^{\prime}\right), \hat{V}_{2}\left(t^{\prime \prime}\right)\right]+\left[\hat{V}_{2}\left(t^{\prime}\right), \hat{V}_{1}\left(t^{\prime \prime}\right)\right]\right| b_{1} b_{2} 0\right\rangle \\
& =-\frac{\wp_{1} \wp_{2}}{2 \hbar^{2}} \int_{t_{0}}^{t} d t^{\prime} \int_{t_{0}}^{t^{\prime}} d t^{\prime \prime}\left[e^{i \omega_{1} \tau\left(t^{\prime}\right)} e^{i \omega_{2} t^{\prime \prime}}\left[\hat{E}\left(t^{\prime}, z_{1}\left(t^{\prime}\right)\right), \hat{E}\left(t^{\prime \prime}, z_{2}\right)\right]+e^{i \omega_{2} t^{\prime}} e^{i \omega_{1} \tau\left(t^{\prime \prime}\right)}\left[\hat{E}\left(t^{\prime}, z_{2}\right), \hat{E}\left(t^{\prime \prime}, z_{1}\left(t^{\prime \prime}\right)\right)\right]\right]
\end{aligned}
$$

Using

$$
\left[\hat{E}\left(t^{\prime}, z_{1}\right), \hat{E}\left(t^{\prime \prime}, z_{2}\right)\right]=\frac{i c \hbar}{2 \epsilon_{0} A_{\mathrm{ph}}} \frac{\partial}{\partial z_{2}}\left(\delta\left[t^{\prime}-t^{\prime \prime}-\left(z_{1}-z_{2}\right) / c\right]-\delta\left[t^{\prime}-t^{\prime \prime}+\left(z_{1}-z_{2}\right) / c\right]\right),
$$

the matrix element becomes

$$
\begin{aligned}
\mathcal{A}_{\mathrm{com}}= & -\frac{i \wp_{1} \wp_{2}}{4 \epsilon_{0} \hbar A_{\mathrm{ph}}} \int_{t_{0}}^{t} d t^{\prime} \int_{t_{0}}^{t^{\prime}} d t^{\prime \prime} e^{i \omega_{1} \tau\left(t^{\prime}\right)} e^{i \omega_{2} t^{\prime \prime}} \frac{\partial}{\partial z_{2}}\left(\delta\left[t^{\prime}-t^{\prime \prime}-\left(z_{1}^{\prime}-z_{2}\right) / c\right]-\delta\left[t^{\prime}-t^{\prime \prime}+\left(z_{1}^{\prime}-z_{2}\right) / c\right]\right) \\
& -e^{i \omega_{2} t^{\prime}} e^{i \omega_{1} \tau\left(t^{\prime \prime}\right)} \frac{\partial}{\partial z_{2}}\left(\delta\left[t^{\prime}-t^{\prime \prime}-\left(z_{2}-z_{1}^{\prime \prime}\right) / c\right]-\delta\left[t^{\prime}-t^{\prime \prime}+\underset{\text { (iiv) }}{\left.\left.\left(z_{2}-z_{1}^{\prime \prime}\right) / c\right]\right)}\right)\right.
\end{aligned}
$$

The second and third delta functions give zero contribution because their arguments are always positive. The first term yields

$$
-\frac{i \wp_{1} \wp_{2}}{4 \epsilon_{0} \hbar A_{\mathrm{ph}}} \frac{\partial}{\partial z_{2}} \int_{-\infty}^{\infty} d t^{\prime} e^{i \omega_{1} \tau\left(t^{\prime}\right)} e^{i \omega_{2}\left[t^{\prime}-\left(z_{1}^{\prime}-z_{2}\right) / c\right]} .
$$

The fourth delta function has $z_{1}^{\prime \prime}=z_{1}\left(t^{\prime \prime}\right)$ in the argument. This makes the integral over $t^{\prime \prime}$ difficult. We resolve this issue by noting that we may extend the integral over $t^{\prime \prime}$ to $t$ by adding $\int_{t^{\prime}}^{t}$ which is zero because the fourth delta function vanishes for $t^{\prime \prime}>t^{\prime}$. Thus we may write the fourth integral as $\int_{t_{0}}^{t} d t^{\prime} \int_{t_{0}}^{t} d t^{\prime \prime}$ and do the integral over $t^{\prime}$ first (which goes with $\left.z_{2}\left(t^{\prime}\right)=z_{2}\right)$ to yield

$$
\frac{i c \wp_{1} \wp_{2}}{4 \epsilon_{0} \hbar A_{\mathrm{ph}}} \frac{\partial}{\partial z_{2}} \int_{t_{0}}^{t} d t^{\prime \prime} e^{i \omega_{1} \tau\left(t^{\prime \prime}\right)} e^{i \omega_{2}\left[t^{\prime \prime}-\left(z_{2}-z_{1}\left(t^{\prime \prime}\right)\right) / c\right]}
$$

Extending to $t_{0} \rightarrow-\infty$ and $t \rightarrow \infty$, we finally obtain

$$
\begin{aligned}
\left\langle a_{1} a_{2} 0|\hat{U}(\infty)| b_{1} b_{2} 0\right\rangle= & -\frac{\wp_{1} \wp_{2} \omega_{2}}{4 \epsilon_{0} \hbar c A_{\mathrm{ph}}} \int_{-\infty}^{\infty} d t^{\prime}\left[e^{i\left(\omega_{1} \tau\left(t^{\prime}\right)+\omega_{2} t^{\prime}\right)-i \omega_{2}\left(z_{1}^{\prime}-z_{2}\right) / c}\right. \\
& +e^{i\left(\omega_{1} \tau\left(t^{\prime}\right)+\omega_{2} t^{\prime}\right)+i \omega_{2}\left(z_{1}^{\prime}-z_{2}\right) / c} \\
& +e^{i\left(\omega_{1} \tau\left(t^{\prime}\right)+\omega_{2} t^{\prime}\right)+i \omega_{2}\left(z_{1}^{\prime}-z_{2}\right) / c} \\
& \left.-e^{i\left(\omega_{1} \tau\left(t^{\prime}\right)+\omega_{2} t^{\prime}\right)-i \omega_{2}\left(z_{1}^{\prime}-z_{2}\right) / c}\right]
\end{aligned}
$$

(i, anticomm-right, $K_{1}$ )

(ii, anticomm-left, $K_{2}$ ) (iv, comm-left, $K_{3}$ ) (iii, comm-right, $K_{4}$ ) 
where $z_{1}^{\prime}$ is $z_{1}\left(t^{\prime}\right)$. So we see that the right-propagating modes cancel while the left-propagating modes add. The net result is

$$
\left\langle a_{1} a_{2} 0|\hat{U}(\infty)| b_{1} b_{2} 0\right\rangle=-\frac{\wp_{1} \wp_{2} \omega_{2}}{2 \epsilon_{0} \hbar c A_{\mathrm{ph}}} \int_{-\infty}^{\infty} d t^{\prime} e^{i\left(\omega_{1} \tau\left(t^{\prime}\right)+\omega_{2} t^{\prime}\right)+i \omega_{2}\left(z_{1}\left(t^{\prime}\right)-z_{2}\right) / c}
$$

\section{APPENDIX B: ALTERNATIVE DERIVATION OF EXCITATION PROBABILITY}

In the interaction representation, the atom-field interaction Hamiltonian reads

$$
\hat{V}(t)=\wp_{2}\left(\hat{\sigma}_{2} e^{-i \omega_{2} t}+\hat{\sigma}_{2}^{\dagger} e^{i \omega_{2} t}\right) \frac{\partial \hat{A}\left(t, z_{2}\right)}{\partial t}+\wp_{1}\left(\hat{\sigma}_{1} e^{-i \omega_{1} \tau}+\hat{\sigma}_{1}^{\dagger} e^{i \omega_{1} \tau}\right) \frac{\partial \hat{A}\left(t, z_{1}\right)}{\partial t},
$$

where index 1 designates the accelerated detector, with proper time along the path of that detector given by $\tau$, and with $\tau$ evaluated at time $t$.

The amplitude that, given the detectors both start out in the ground state $\left|b_{1} b_{2} 0\right\rangle$ at time $-T$ and end up in their excited state $\left|a_{1} a_{2} 0\right\rangle$ at time $t=T$, to lowest order in the coupling dipole moment $\wp_{1,2}$ is

$$
\begin{aligned}
\mathcal{A} & =-\left\langle a_{1} a_{2} 0\left|\int_{-T}^{T} d t \int_{-T}^{t} d t^{\prime} \hat{V}(t) \hat{V}\left(t^{\prime}\right)\right| b_{1} b_{2} 0\right\rangle \\
& =-\wp_{1} \wp_{2}\left\langle 0\left|\int_{-T}^{T} d t \int_{-T}^{T} d t^{\prime} \theta\left(t-t^{\prime}\right)\left[e^{i \omega_{2} t} \frac{\partial \hat{A}\left(t, z_{2}\right)}{\partial t} e^{i \omega_{1} \tau\left(t^{\prime}\right)} \frac{\partial \hat{A}\left(t^{\prime}, z_{1}\right)}{\partial t^{\prime}}+e^{i \omega_{1} \tau(t)} \frac{\partial \hat{A}\left(t, z_{1}\right)}{\partial t} e^{i \omega_{2} t^{\prime}} \frac{\partial \hat{A}\left(t^{\prime}, z_{2}\right)}{\partial t^{\prime}}\right]\right| 0\right\rangle,
\end{aligned}
$$

where $\theta(x)$ is the Heaviside function which is 1 for $x>0$ and 0 for $x<0$. In the following we will also use $\sigma(x)=\theta(x)-\theta(-x)$, the antisymmetric step function (not to be confused with the atomic lowering operator $\hat{\sigma}$.)

The operator $\hat{A}$ is a scalar function, which represents the vector potential in $1+1$ dimensional "electromagnetism", which has the form

$$
\hat{A}\left(t, z_{2}\right)=\int_{0}^{\infty} \frac{1}{\sqrt{4 \pi \omega}}\left[\hat{b}_{R \omega} e^{-i \omega u_{2}}+\hat{b}_{R \omega}^{\dagger} e^{i \omega u_{2}}+\hat{b}_{L \omega} e^{-i \omega v_{2}}+\hat{b}_{L \omega}^{\dagger} e^{i \omega v_{2}}\right] d \omega,
$$

where the $L, R$ designate the left, right moving modes, $u_{2}=t-z_{2} / c, v_{2}=t+z_{2} / c$ with $z_{2}$ the location $\left(z_{2}<0\right)$ of the geodesic detector, and

$$
\hat{A}\left(t, z_{1}\right)=\int_{0}^{\infty} \frac{1}{\sqrt{4 \pi \omega}}\left[b_{R \omega} e^{-i \omega u_{1}}+b_{R \omega}^{\dagger} e^{i \omega u_{1}}+b_{L \omega} e^{-i \omega v_{1}}+b_{L \omega}^{\dagger} e^{i \omega v_{1}}\right] d \omega,
$$

where $u_{1}=t-z_{1} / c=-c e^{-a \tau / c} / a$ and $v_{1}=t+z_{1} / c=c e^{a \tau / c} / a$.

The field operator $\hat{A}$ can be written as a sum of left and right moving terms,

$$
\hat{A}(t, z)=A_{R}(u(t, z))+\hat{A}_{L}(v(t, z)) .
$$

Also if $\xi$ is a function of $\chi$, then $\partial_{\xi} \hat{A}(\xi) d \xi=\partial_{\chi} \hat{A}(\chi) d \chi$ for any function $\hat{A}$ of variables $\xi, \chi=\chi(\xi)$.

We can write the expression for the probability amplitude $\mathcal{A}$ in terms of the $R, L$ terms, and obtain

$$
\begin{aligned}
\mathcal{A}_{R}= & -\wp_{1} \wp_{2} \int_{u_{2}(-T)}^{u_{2}(T)} d u_{2} \int_{u_{1}(-T)}^{u_{1}(T)} d u_{1} e^{i \omega_{2} t\left(u_{2}\right)} e^{i \omega_{1} \tau\left(u_{1}\right)} \\
& \left\langle 0\left|\partial_{u_{1}}\left(\theta\left(t\left(u_{2}\right)-t\left(u_{1}\right)\right) \partial_{u_{2}} \hat{A}_{R}\left(u_{2}\right) \hat{A}_{R}\left(u_{1}\right)+\theta\left(t\left(u_{1}\right)-t\left(u_{2}\right)\right) \hat{A}_{R}\left(u_{1}\right) \partial_{u_{2}} \hat{A}_{R}\left(u_{2}\right)\right)\right| 0\right\rangle
\end{aligned}
$$

and similarly for $\mathcal{A}_{L}$ by replacing $u$ by $v$ and $R$ by $L$ in the above.

Now, we can write

$$
\begin{array}{r}
\theta\left(t\left(u_{2}\right)-t\left(u_{1}\right)\right) \partial_{u_{2}} \hat{A}_{R}\left(u_{2}\right) \hat{A}_{R}\left(u_{1}\right)+\theta\left(t\left(u_{1}\right)-t\left(u_{2}\right)\right) \hat{A}_{R}\left(u_{1}\right) \partial_{u_{2}} \hat{A}_{R}\left(u_{2}\right) \\
\quad=\frac{1}{2}\left(\left\{\partial_{u_{2}} \hat{A}_{R}\left(u_{2}\right), \hat{A}_{R}\left(u_{1}\right)\right\}+\sigma\left(t\left(u_{2}\right)-t\left(u_{1}\right)\right)\left[\partial_{u_{2}} \hat{A}_{R}\left(u_{2}\right), \hat{A}_{R}\left(u_{1}\right)\right]\right)
\end{array}
$$

where $\{$,$\} is the anticommutator while [,] is the commutator. We also get the same for the L$ terms by replacing $R$ by $L$ and $u$ by $v$.

Taking the vacuum expectation values of the above operators, we have

$$
\begin{aligned}
& \left\langle 0\left|\frac{1}{2}\left(\left\{\partial_{u_{2}} \hat{A}_{R}\left(u_{2}\right), \hat{A}_{R}\left(u_{1}\right)\right\}+\sigma\left(t\left(u_{2}\right)-t\left(u_{1}\right)\right)\left[\partial_{u_{2}} \hat{A}_{R}\left(u_{2}\right), \hat{A}_{R}\left(u_{1}\right)\right]\right)\right| 0\right\rangle \\
& \quad=\frac{i}{8 \pi}\left[\int_{0}^{\infty}\left(e^{i \omega\left(u_{2}-u_{1}\right)}-e^{-i \omega\left(u_{2}-u_{1}\right)}\right) d \omega+\sigma\left(t\left(u_{2}\right)-t\left(u_{1}\right)\right) \int_{0}^{\infty}\left(e^{i \omega\left(u_{2}-u_{1}\right)}+e^{-i \omega\left(u_{2}-u_{1}\right)}\right) d \omega\right] \\
& \quad=\frac{1}{8 \pi}\left[\lim _{\lambda \rightarrow 0^{+}}\left(\frac{1}{\left(u_{2}-u_{1}\right)-i \lambda}+\frac{1}{\left(u_{2}-u_{1}\right)+i \lambda}\right)+2 \pi i \sigma\left(t\left(u_{2}\right)-t\left(u_{1}\right)\right) \delta\left(u_{2}-u_{1}\right)\right] .
\end{aligned}
$$


Now the term multiplying the $\sigma\left(t\left(u_{2}\right)-t\left(u_{1}\right)\right)$ is nonzero only when $u_{2}=u_{1}$, and since the accelerated curve points always lie to the right of the geodesic points, $t\left(u_{1}\right)$ is greater than $t\left(u_{2}\right)$, and $\sigma\left(t\left(u_{2}\right)-t\left(u_{1}\right)\right)=-1$ and

$$
\begin{aligned}
\langle 0| & \frac{1}{2}\left(\left\{\partial_{u_{2}} \hat{A}_{R}\left(u_{2}\right), \hat{A}_{R}\left(u_{1}\right)\right\}+\sigma\left(t\left(u_{2}\right)-t\left(u_{1}\right)\right)\left[\partial_{u_{2}} \hat{A}_{R}\left(u_{2}\right), \hat{A}_{R}\left(u_{1}\right)\right]\right)|0\rangle \\
= & \frac{1}{8 \pi}\left[\lim _{\lambda \rightarrow 0^{+}}\left(\frac{1}{\left(u_{2}-u_{1}\right)-i \lambda}+\frac{1}{\left(u_{2}-u_{1}\right)+i \lambda}\right)-2 \pi i \delta\left(u_{2}-u_{1}\right)\right] .
\end{aligned}
$$

Similarly for the left traveling terms

$$
\begin{aligned}
\langle 0| & \frac{1}{2}\left(\left\{\partial_{v_{2}} \hat{A}_{L}\left(v_{2}\right), \hat{A}_{L}\left(v_{1}\right)\right\}+\sigma\left(t\left(v_{2}\right)-t\left(v_{1}\right)\right)\left[\partial_{v_{2}} \hat{A}_{L}\left(v_{2}\right), \hat{A}_{L}\left(v_{1}\right)\right]\right)|0\rangle \\
= & \frac{1}{8 \pi}\left[\lim _{\lambda \rightarrow 0^{+}}\left(\frac{1}{\left(v_{2}-v_{1}\right)-i \lambda}+\frac{1}{\left(v_{2}-v_{1}\right)+i \lambda}\right)+2 \pi i \delta\left(v_{2}-v_{1}\right)\right]
\end{aligned}
$$

because $\sigma\left(t\left(v_{2}\right)-t\left(v_{1}\right)\right)=+1$ since the left moving null curve goes from the accelerated curve in the past to the unaccelerated curve in the future.

Thus we have

$$
\mathcal{A}_{R}=-\frac{\wp_{1} \wp_{2}}{8 \pi} \lim _{\lambda \rightarrow 0} \int_{-\infty}^{0} d u_{1} \int_{-\infty}^{\infty} d u_{2} \partial_{u_{1}} e^{i \omega_{2} u_{2}}\left[\frac{1}{\left(u_{2}-u_{1}\right)-i \lambda}+\frac{1}{\left(u_{2}-u_{1}\right)+i \lambda}-2 \pi i \delta\left(u_{2}-u_{1}\right)\right] e^{i \omega_{1} \tau\left(u_{1}\right)}=0,
$$

while

$$
\begin{aligned}
\mathcal{A}_{L} & =-\frac{\wp_{1} \wp_{2}}{8 \pi} \lim _{\lambda \rightarrow 0} \int_{0}^{\infty} d v_{1} \int_{-\infty}^{\infty} d v_{2} \partial_{v_{1}} e^{i \omega_{2} v_{2}}\left[\frac{1}{\left(v_{2}-v_{1}\right)-i \lambda}+\frac{1}{\left(v_{2}-v_{1}\right)+i \lambda}+2 \pi i \delta\left(v_{2}-v_{1}\right)\right] e^{i \omega_{1} \tau\left(v_{1}\right)} \\
& =-\frac{\wp_{1} \wp_{2} \omega_{2}}{2} \int_{0}^{\infty} e^{i \omega_{1} \tau\left(v_{1}\right)} e^{i \omega_{2} v_{1}} d v_{1}
\end{aligned}
$$

[1] L. C. B. Crispino, A. Higuchi, and G. E. A. Matsas, The Unruh effect and its applications, Rev. Mod. Phys. 80, 787 (2008).

[2] G. Moore, Quantum theory of the electromagnetic field in a variable-length one-dimensional cavity, J. Math. Phys. 11, 2679 (1970).

[3] S. A. Fulling, Nonuniqueness of canonical field quantization in riemannian space-time, Phys. Rev. D 7, 2850 (1973).

[4] S. W. Hawking, Black hole explosions?, Nature (London) 248 , 30 (1974); Particle creation by black holes, Commun. Math. Phys. 43, 199 (1975).

[5] D. N. Page, Particle emission rates from a black hole: Massless particles from an uncharged, nonrotating hole, Phys. Rev. D 13, 198 (1976).

[6] W. G. Unruh, Notes on black hole evaporation, Phys. Rev. D 14, 870 (1976).

[7] A. O. Barut and J. P. Dowling, Quantum electrodynamics based on self-fields: On the origin of thermal radiation detected by an accelerating observer, Phys. Rev. A 41, 2277 (1990).
[8] J. Audretsch and R. Müller, Localized discussion of stimulated processes for Rindler observers and accelerated detectors, Phys. Rev. D 49, 4056 (1994).

[9] M. O. Scully, V. V. Kocharovsky, A. Belyanin, E. Fry, and F. Capasso, Enhancing Acceleration Radiation from Ground-State Atoms Via Cavity Quantum Electrodynamics, Phys. Rev. Lett. 91, 243004 (2003)

[10] W. G. Unruh and R. M. Wald, What happens when an accelerating observer detects a Rindler particle, Phys. Rev. D 29, 1047 (1984).

[11] The electric field per photon in the atomic frame is actually given by $\epsilon_{k} \exp (-a \tau / c)$, see Ref. [9]. However, this is not an important point for present purposes, and for simplicity we will not include such complications in the calculation of Sec. II and Appendix A. The treatment of Appendix B does however include such factors. The quantization volume is $V_{k}=$ $A_{k} L$, where $A_{k}$ is the mode area and $L$ is the corresponding length. 